\title{
Creating mosquito-free outdoor spaces using transfluthrin-treated chairs and ribbons
}

\author{
John P. Masalu ${ }^{1,2 *}$, Marceline Finda ${ }^{1,3}$, Gerry F. Killeen ${ }^{1,4}$, Halfan S. Ngowo ${ }^{1,5}$, Polius G. Pinda ${ }^{1}$ \\ and Fredros O. Okumu $u^{1,2,3,5}$
}

\begin{abstract}
Background: Residents of malaria-endemic communities spend several hours outdoors performing different activities, e.g. cooking, story-telling or eating, thereby exposing themselves to potentially-infectious mosquitoes. This compromises effectiveness of indoor interventions, notably long-lasting insecticide-treated nets (LLINs) and indoor residual spraying (IRS). This study characterized common peri-domestic spaces in rural south-eastern Tanzania, and assessed protective efficacy against mosquitoes of hessian fabric mats and ribbons treated with the spatial repellent, transfluthrin, and fitted to chairs and outdoor kitchens, respectively.
\end{abstract}

Methods: Two hundred households were surveyed, and their most-used peri-domestic spaces physically characterized. Protective efficacies of locally-made transfluthrin-emanating chairs and hessian ribbons were tested in outdoor environments of 28 households in dry and wet seasons, using volunteer-occupied exposure-free double net traps. CDC light traps were used to estimate host-seeking mosquito densities within open-structure outdoor kitchens. Fieldcollected Anopheles arabiensis and Anopheles funestus mosquitoes were exposed underneath the chairs to estimate $24 \mathrm{~h}$-mortality. Finally, The World Health Organization insecticide susceptibility tests were conducted on wild-caught Anopheles from the villages.

Results: Approximately half (52\%) of houses had verandas. Aside from these verandas, most houses also had peridomestic spaces where residents stayed most times ( $67 \%$ of houses with verandas and $94 \%$ of non-veranda houses). Two-thirds of these spaces were sited under trees, and only one third (34.4\%) were built-up. The outdoor structures were usually makeshift kitchens having roofs and partial walls. Transfluthrin-treated chairs reduced outdoor-biting An. arabiensis densities by $70-85 \%$, while transfluthrin-treated hessian ribbons fitted to the outdoor kitchens caused 77-81\% reduction in the general peri-domestic area. Almost all the field-collected An. arabiensis (99.4\%) and An. funestus (100\%) exposed under transfluthrin-treated chairs died. The An. arabiensis were susceptible to non-pyrethroids (pirimiphos methyl and bendiocarb), but resistant to pyrethroids commonly used on LLINs (deltamethrin and permethrin).

Conclusion: Most houses had actively-used peri-domestic outdoor spaces where exposure to mosquitoes occurred. The transfluthrin-treated chairs and ribbons reduced outdoor-biting malaria vectors in these peri-domestic spaces, and also elicited significant mortality among pyrethroid-resistant field-caught malaria vectors. These two new prototype formats for transfluthrin emanators, if developed further, may constitute new options for complementing LLINs and IRS with outdoor protection against malaria and other mosquito-borne pathogens in areas where peri-domestic human activities are common.

\footnotetext{
*Correspondence: jpaliga@ihi.or.tz

${ }^{1}$ Environmental Health and Ecological Sciences Department, Ifakara

Health Institute, Morogoro, United Republic of Tanzania

Full list of author information is available at the end of the article
}

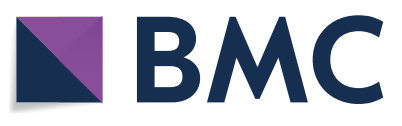

(c) The Author(s) 2020. This article is licensed under a Creative Commons Attribution 4.0 International License, which permits use, sharing, adaptation, distribution and reproduction in any medium or format, as long as you give appropriate credit to the original author(s) and the source, provide a link to the Creative Commons licence, and indicate if changes were made. The images or other third party material in this article are included in the article's Creative Commons licence, unless indicated otherwise in a credit line to the material. If material is not included in the article's Creative Commons licence and your intended use is not permitted by statutory regulation or exceeds the permitted use, you will need to obtain permission directly from the copyright holder. To view a copy of this licence, visit http://creativeco mmons.org/licenses/by/4.0/. The Creative Commons Public Domain Dedication waiver (http://creativecommons.org/publicdomain/ zero/1.0/) applies to the data made available in this article, unless otherwise stated in a credit line to the data. 
Keywords: Peri-domestic spaces, Transfluthrin-treated chairs, Eave ribbons, Transfluthrin, Spatial repellents, Outdoorbiting, Malaria vectors, Ifakara Health Institute

\section{Background}

Since 2000, malaria morbidity and mortality have tremendously declined in sub-Saharan Africa [1-4], though the recent evidence suggests that such gains are starting to stagnate [3-5]. Most of the gains observed between 2000 and 2015 were estimated to have been contributed by the existing core indoor vector control interventions, i.e. insecticide-treated nets (ITNs) and indoor residual spraying (IRS) [2, 6-8]. Long-lasting insecticide-treated nets (LLINs) and IRS are effective against indoor-biting and indoor-resting mosquitoes, but are less effective against outdoor-biting mosquitoes, which are important vectors of residual malaria transmission [9-12]. It has been estimated that the Anopheles bites not preventable by LLINs could be causing up to 10 million additional malaria cases annually [12]. As a result, LLINs and IRS require complimentary interventions to achieve the 2030 global targets of reducing malaria burden by at least $90 \%$ and elimination in 35 endemic countries [13].

In many malaria-endemic communities, people spend several hours cooking, eating and socializing outdoors in the early evenings before they go to sleep, and also in the early mornings after they wake up [14], when malaria vectors may be active and mediating transmission [11]. Some of these outdoor activities, as well as sleeping outdoors [15], are partly attributable to warm climate [16], but they also have strong cultural determinants [17]. The importance of outdoor malaria transmission, and associated outdoor human activities, are now well-established $[9,10,14,17]$. However, there are still gaps regarding appropriate interventions to address these gaps. The characteristics of the peri-domestic spaces where households conduct outdoor activities remain poorly documented, despite being essential for designing, creating and testing interventions to complement LLINs and IRS by protecting such outdoor spaces.

Several intervention options have been proposed as candidates for closing these malaria transmission gaps [18]. Examples include: (a) outdoor-baited traps [19, 20], (b) attractive targeted sugar baits [21], (c) pyrethroidtreated clothing [22, 23], zooprophylaxis [24] and repellents [25] among others. Topical repellents applied on human skin are widely available for personal protection in some areas. However, commercial formulations of government-sectioned scale-up campaigns of such topical repellents are limited because they protect only individual users [26], have low user compliance rates and acceptance [27-29], and have only short-term efficacy
[30]. They are also expensive for repeated use by the lowincome populations at greatest risk.

In contrast, spatial repellents are volatile insecticides that diffuse into the air as vapour, and may protect multiple people within the surrounding space against outdoor-biting malaria vectors [31-35]. In recent years, several versions and delivery formats have been developed, which allow wide-area protection of multiple persons without repeated application for several months [31-34, 36, 37]. In particular, a wide range of transfluthrin emanator prototypes based on treated hessian fabric products have been recently developed that protect indoor and outdoor spaces for several months without repeated reapplication [31-34, 36, 37]. Transfluthrin also has additional properties beyond just spatial repellency that include toxicity to mosquitoes, and incapacitation that prevents blood-feeding, which could contribute to community-wide mass effects, even for non-users [37, 38].

Improved understanding of the peri-domestic spaces coupled with new interventions that can be effective in such spaces, could potentially address current challenges related with exposure to outdoor-biting exposure and transmission risk. This study was, therefore, aimed at addressing two key knowledge gaps by: (a) characterizing the common peri-domestic spaces used by communities in rural south-eastern Tanzania for various outdoor activities, and, (b) assessing the protective efficacies of two recently-developed hessian-based transfluthrinemanator prototypes, specifically transfluthrin-treated chairs and transfluthrin-treated hessian ribbons wrapped around outdoor kitchens, against outdoor-biting malaria vectors and other pathogens-carrying mosquitoes in those peri-domestic spaces.

\section{Methods}

\section{Study area}

The study was implemented in Lupiro village $\left(8.385^{\circ} \mathrm{S}\right.$, $36.670^{\circ}$ E) (Fig. 1), in the Kilombero valley, south-eastern Tanzania. Households were selected from four subvillages namely: (a) Ndoro; (b) Libaratula; (c) Mabatini and (d) Lupiro Kati. Most residents here were peasants, cultivating rice, maize and other crops. Houses have brick or mud walls, and metal (corrugated iron sheets) or grass-thatched roofs. Annual rainfall is $1200-1600 \mathrm{~mm}$, and temperatures range between 20.0 and $32.6{ }^{\circ} \mathrm{C}$ [39, 40]. Principal malaria vectors in this area are Anopheles funestus and Anopheles arabiensis with the former 


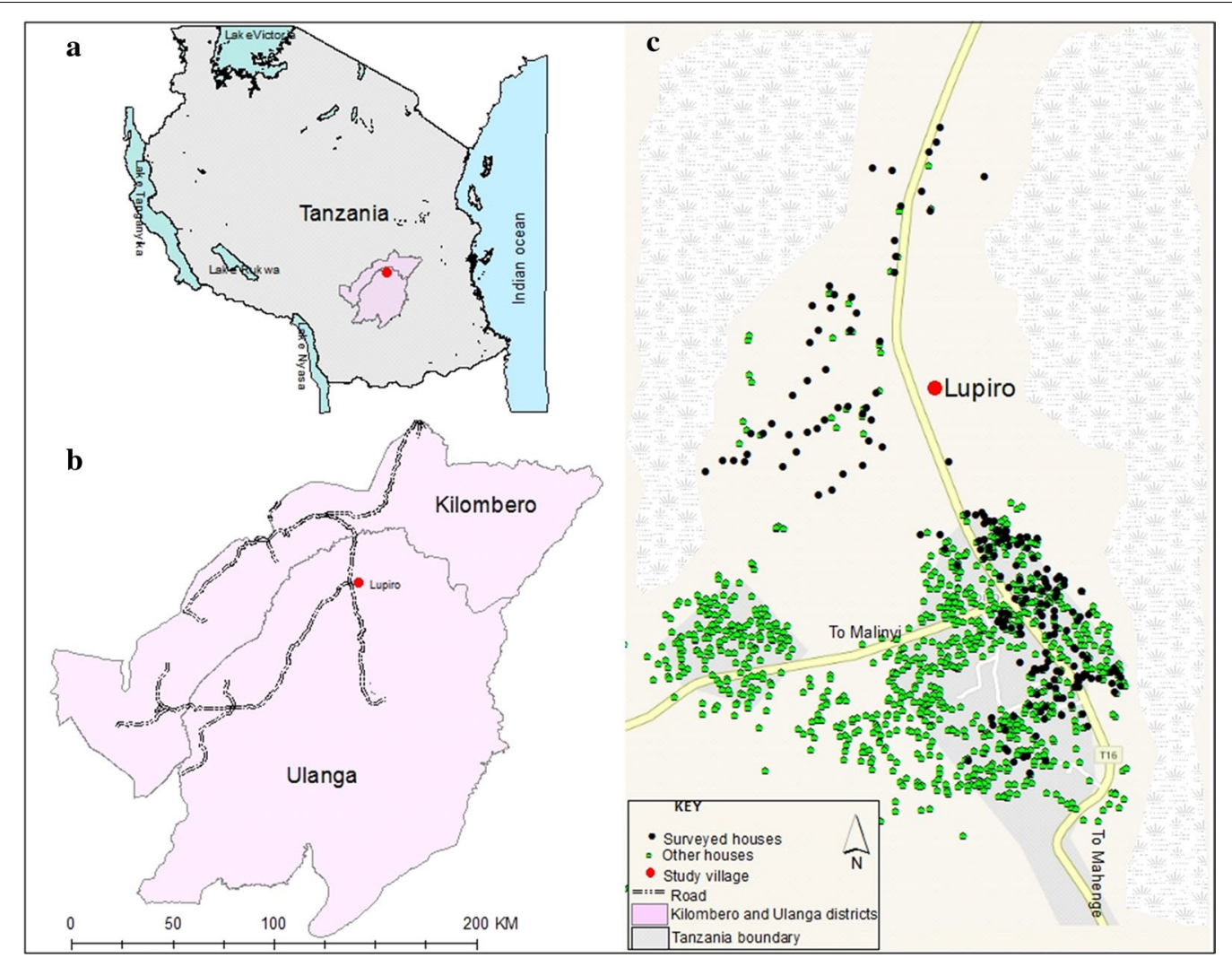

Fig. 1 Illustration of the location of Ulanga and Kilombero districts in the map of Tanzania (a), the location of Lupiro village in Ulanga district (b) and household location in Lupiro village showing both surveyed and those did not (c)

contributing over $80 \%$ of transmission [41]. Both $A n$. arabiensis and An. funestus populations in the area have been shown to be resistant to multiple public health insecticides including pyrethroids, carbamates and organochlorides [41-43]. LLINs are the main malaria prevention method, most of which are distributed by the government [44].

\section{Characterization of the peri-domestic spaces}

Two hundred (200) households were surveyed, including 50 from each sub-village (Fig. 1), selected via stratified random sampling. Data were collected using electronic tablets using KoboCollect ${ }^{\mathrm{TM}}$, an open access software programmed using Open Data Kit (ODK) [45]. Trained research teams were assigned to each sub-village. Written informed consent was obtained from each of the 200 households. For each household, the peri-domestic spaces were observed directly to characterize them physically based on use, physical site and whether they were built-up or not. Digital pictures were taken of the different peri-domestic environments. The research team also administered survey questions to the household heads to capture: (a) identification information such as age, (b) education level, (c) socio-economic data including source of income, possession of radio, television, cell phone among others, (d) information on peri-domestic spaces such as presence of other peridomestic spaces apart from veranda, and (e) their usage, presence of peri-domestic spaces if the house had no veranda and their usage.

The peri-domestic spaces were classified as either: (a) built-up spaces attached to the main houses, i.e. veranda extensions; (b) built-up spaces not attached to the main houses, e.g. separate kitchens, and (c) non-built-up or other peri-domestic spaces commonly used for various outdoor activities. The outdoor built up structures were also characterized based on the roofing and wall types.

\section{Transfluthrin-treated chairs and hessian ribbons}

For the dry season experiment, six identical chairs made of wood and metal frame were constructed by a local carpenter while for the wet season experiment 15 chairs were made (Fig. 2a, b). The chairs were fitted underneath with four standardized hessian fabric mats: two measuring $42 \mathrm{~cm} \times 43 \mathrm{~cm}$ and fitted underneath the right and left sides of the chair and other two measuring $20 \mathrm{~cm} \times 33 \mathrm{~cm}$, which were fitted underneath the middle 

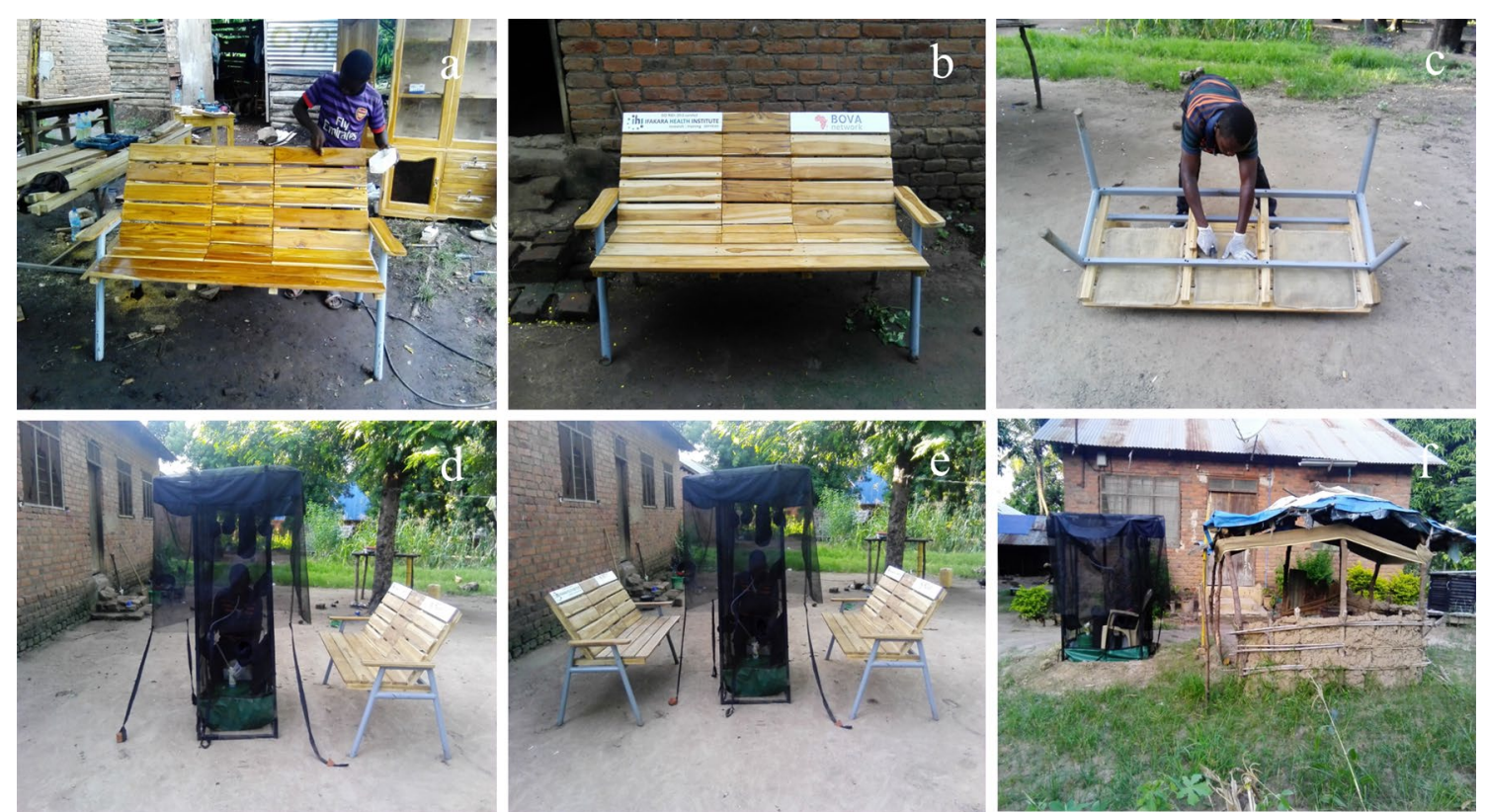

Fig. 2 Design and prototyping of the wooden chairs at the local carpentry (a), overview of the prototyped chair (b), fitting transfluthrin-treated hessian mat underneath the chair (c), one transfluthrin-treated chair with the DN-Mini trap positioned $0.5 \mathrm{~m}$ (d), two transfluthrin-treated chairs with DN-Mini trap installed $0.5 \mathrm{~m}(\mathbf{e})$; and outdoor kitchen fitted with transfluthrin-treated sisal ribbon with DM-Mini trap positioned $1.2 \mathrm{~m}$ (f)

part of the chair (Fig. 2c). These mats were made by a local seamstress at the Ifakara Health Institute fabrication facility (the MozzieHouse). The hessian mats had been treated in emulsified solutions containing $2 \%$ transfluthrin (Bayer AG, Germany), prepared as previously described [31,33].

Similarly, the hessian ribbons were prepared as previously described by Mmbando et al. [36]. Each ribbon had $15 \mathrm{~cm}$ width and $10 \mathrm{~m}$ length, and were also made locally at the MozzieHouse. More detailed descriptions of the hessian ribbons have previously been published by Ogoma et al. [31] and Mmbando et al. [36]. The ribbons were also treated in a $2 \%$ emulsified solution of transfluthrin as previously described [36].

\section{Assessing protective efficacies of transfluthrin-treated chairs and ribbons}

This assessment was conducted in two seasons: dry and wet seasons, between September to October 2019 and between January to February 2020 as dry and wet seasons, respectively. Following the characterization of the peri-domestic spaces as described above, eight households with outdoor kitchens were selected for a smallscale assessment of protective efficacies of the two candidate interventions in the dry season. The houses were paired and assigned as follows: (a) a control arm, where neither transfluthrin-treated chairs nor transfluthrin-treated ribbons were used, (b) a treatment arm where one transfluthrin-treated chair was used, (c) a second treatment arm where two transfluthrin-treated chairs were used, and (d) a third treatment arm where transfluthrin-treated hessian ribbons were used around the outdoor kitchens. In each arm, two houses were enrolled.

One consenting adult male volunteer was assigned to each household, to sit inside the exposure-free miniaturized double nets trap (DN-Mini) [46] from 1900 to $2300 \mathrm{~h}$. The volunteer spent $45 \mathrm{~min}$ each hour retrieving all host-seeking mosquitoes caught in the DN-Mini while attempting to bite him. For the households with transfluthrin-emanating chairs, the DN-Mini was installed $0.5 \mathrm{~m}$ from the chairs (Fig. 2d, e). For households with transfluthrin-treated hessian ribbons, the ribbon was fitted $1.3 \mathrm{~m}$ above ground (Fig. 2f) onto the outdoor kitchens. CDC light traps [47] were suspended inside these makeshift kitchens to collect host-seeking mosquitoes nightly, while DN-Mini traps were set beside the kitchens to assess biting risk in the general peri-domestic space (Fig. 2f).

Each treatment arm was initially located in two houses per experimental night, but was rotated between the houses using a $4 \times 4$ Latin square design over 32 experimental nights, so that each treatment or control arm was tested at each of the eight houses four times. The primary outcome was number of mosquitoes of different species caught in the DN-Mini or the CDC light traps per house 
per night. All treated materials were carefully shifted between the houses to avoid any contamination during the rotations. As the experiments were conducted outdoors with enough airflow, there was no need to break for wash out. Instead, a control set up was used to monitor mortality of mosquitoes as described in the sub-section below. Each morning the collected mosquitoes were sorted and identified using morphological keys [48]. In the wet season, 20 households were enrolled making five households in each arm for other 32 nights. The same procedure was adopted as described in dry season.

\section{Assessing mortality effects of the transfluthrin-treated chairs on mosquitoes}

This assay was done using three different groups of mosquitoes, as follows: (a) field-collected An. arabiensis and $A n$. funestus of unknown age, which are known to be pyrethroid resistant in this setting [41-43], (b) laboratory-reared $A n$. arabiensis from a pyrethroid-susceptible colony of local origin, and (c) laboratory-reared Aedes aegypti from a pyrethroid-susceptible colony of local origin [49].

The wild-caught $A n$. arabiensis females were collected using a separate set of eight DN-Mini traps [46] set outdoors at households without any transfluthrin treatments. Eight consenting adult male volunteers were involved in these collections each night from $1900 \mathrm{~h}$ to 0100 h. As population densities of An. funestus in this study area were very low, CDC light traps were used to collect adult females of this species from another village (Tulizamoyo $(-8.3669,36.7336)$ ) approximately $30 \mathrm{~km}$ away.

Each morning captured mosquitoes were sorted and $A n$. arabiensis and $A n$. funestus females separated in two cages containing 100 mosquitoes per species (four cages in total). Since the Anopheles gambiae sensu lato (s.l.) in this area are known to consist exclusively of $A n$. arabiensis [33], no molecular identification was required. Similarly, since indoor collections of An. funestus s.l. have consistently been found to be $>90 \% A n$. funestus sensu stricto [50], it was assumed that these were the dominant species in the collections. The separated mosquitoes were kept at a field insectary (average temperature: $26.75 \pm 0.09{ }^{\circ} \mathrm{C}$; relative humidity: $73.26 \pm 0.46 \%$ ) for acclimatization for at least $20 \mathrm{~h}$ before testing the next evening.

For the tests, two chairs were placed within open verandas of two separate houses. One of the chairs was fitted underneath with transfluthrin-treated hessian mats, while the other was fitted with an untreated hessian mat (control). The caged mosquitoes were placed underneath each chair overnight (1900 h to $0700 \mathrm{~h}$ ). A simple water moat was used to prevent ants from eating the mosquitoes. Each morning, the cages were returned to the field insectary and monitored for further $12 \mathrm{~h}$, totaling $24 \mathrm{~h}$ of observation since start of exposure. This procedure was repeated 10 times (totaling 1140 mosquitoes) for field-collected $A n$. arabiensis and five times (totaling 490 mosquitoes) for field-collected An. funestus tested in control and treated arms.

Similar tests were conducted using cages containing 100 laboratory-reared $A n$. arabiensis or 100 laboratoryreared Ae. aegypti. Since Ae. aegypti mosquitoes are active during the day, they were exposed from 0800 to 1900 h each day, as opposed to the Anopheles mosquitoes, which were exposed at night. Percentage mortality of mosquitoes was calculated for each species separately as a proportion of total exposed.

\section{Testing susceptibility of local malaria vector populations to common public health pesticides}

In order to determine phenotypic resistance status of local mosquito populations to common pesticides, standard discriminatory tests were performed using standard WHO susceptibility bioassays [51]. Since transfluthrin is a pyrethroid, the tests also provided indication of how the transfluthrin-based interventions evaluated here (transfluthrin-treated chairs and transfluthrin-treated hessian ribbons) evaluated here would perform against wild pyrethroid-resistant mosquito populations. The susceptibility tests were done for: (a) $0.1 \%$ bendiocarb, a carbamate; (b) $4.0 \%$ dichlorodiphenyltrichloroethane (DDT), an organochloride; (c) $0.25 \%$ pirimiphos methyl, an organophosphate, (d) $0.75 \%$ permethrin, a type I pyrethroid; and (e) $0.05 \%$ deltamethrin, a type II pyrethroid.

Female $A n$. arabiensis mosquitoes were collected from nearby rice fields as larvae, and reared to emergence at Ifakara Health Institute vector biology laboratory, the VectorSphere. The susceptibility tests were done using 3-day old adult females, using at least 100 mosquitoes per test (25 per replicate), with at least 4 replicates as described in the recent WHO guidelines [51].

\section{Data analysis}

The survey data was summarized in ODK analysis module [45] to generate descriptive statistics of peridomestic spaces and their usage. Data on efficacy of the transfluthrin-treated chairs and ribbons was analysed using $\mathrm{R}$ open-source statistical software [52], primarily using generalized linear mixed-effects models [53], each time modelling the numbers of mosquitoes of a given species caught as a function of the treatments (fixed factors), and fitting the data onto Poisson distributions. Volunteer ID, day and house ID were included as random factors in the models. 
Table 1 Characteristics of the study participants and their houses in 200 surveyed households in Lupiro village, Ulanga District, south-eastern Tanzania

\begin{tabular}{llll}
\hline Characteristics & Category & $\begin{array}{l}\text { Total number } \\
\text { surveyed }(\boldsymbol{n})\end{array}$ & Proportion (\%) \\
\hline Gender & Male & 72 & 36.0 \\
& Female & 128 & 64.0 \\
Age & Average & 38.5 & NA \\
Wall type & Bricks & 153 & 76.5 \\
& Mud and stick & 46 & 23.0 \\
\multirow{2}{*}{ Roof type } & Others & 1 & 0.5 \\
& Iron-sheets & 140 & 70 \\
& Thatched & 56 & 28.0 \\
& Others & 4 & 2.0 \\
\hline
\end{tabular}

\section{Results}

Characteristics of households

The demographic characteristics of household heads, and physical characteristics of all the 200 houses visited are summarized in Table 1. Most of the household heads were female $(128 / 200)$. The main construction materials were bricks for the walls (153/200) and corrugated iron sheets for the roofs $(140 / 200)$. Full details are found in Table 1.

\section{Characteristics of the peri-domestic spaces}

Table 2 provides a summary of the physical characteristics of peri-domestic spaces where residents spent time outdoors in the evenings before bedtime. Of the 200 households observed, 52\% (103/200) had built-up

Table 2 Peridomestic space characteristic of the households surveyed in Lupiro village, Ulanga district, south-eastern Tanzania

\begin{tabular}{|c|c|c|c|c|c|}
\hline \multicolumn{3}{|l|}{ Household with veranda $(\mathrm{N}=103)$} & \multicolumn{3}{|l|}{ Household without veranda $(\mathrm{N}=97)$} \\
\hline Characterization & $\mathbf{n}$ & Percentage & Characterization & $\mathbf{n}$ & Percentage \\
\hline Open veranda & 69 & 67.0 & N/A & & \\
\hline Closed veranda & 34 & 33.0 & N/A & & \\
\hline \multicolumn{6}{|l|}{ Usage } \\
\hline Resting & 92 & 42.2 & N/A & & \\
\hline Cooking & 67 & 30.7 & N/A & & \\
\hline Eating & 56 & 25.7 & N/A & & \\
\hline Others & 3 & 1.4 & N/A & & \\
\hline Other peri-domestic space & & & Other peri-domestic space & & \\
\hline Yes & 69 & 67 & Yes & 91 & 93.8 \\
\hline No & 34 & 33 & No & 6 & 6.2 \\
\hline Built structure & 23 & & Built structure & 32 & \\
\hline Roof & 23 & 100 & Roof & 31 & 96.9 \\
\hline No roof & 0 & 0 & No roof & 1 & 3.1 \\
\hline Wall & 7 & 30.4 & Wall & 10 & 31.3 \\
\hline No wall & 16 & 69.6 & No wall & 22 & 68.7 \\
\hline Average distance from the houses (m) & 6.3 & & Average distance from the houses (m) & 6.8 & \\
\hline Usage & & & Usage & & \\
\hline Resting & 9 & 24.3 & Resting & 19 & 29.2 \\
\hline Cooking & 22 & 59.5 & Cooking & 30 & 46.2 \\
\hline Eating & 6 & 16.2 & Eating & 16 & 24.6 \\
\hline Non-built structure & 46 & & Non-built structure & 59 & \\
\hline Under the tree & 34 & 62.9 & Under the tree & 28 & 42.4 \\
\hline Open space & 19 & 35.2 & Open space & 34 & 51.5 \\
\hline Others & 1 & 1.9 & Others & 4 & 6.1 \\
\hline Average distance from the houses (m) & 6.8 & & Average distance from the houses (m) & 6.2 & \\
\hline Usage & & & Usage & & \\
\hline Resting & 32 & 43.2 & Resting & 54 & 38.0 \\
\hline Cooking & 20 & 27.0 & Cooking & 48 & 33.8 \\
\hline Eating & 22 & 29.7 & Eating & 40 & 28.2 \\
\hline
\end{tabular}

$n$ total number of peridomestic space characterized, $N / A$ not required 
veranda (Fig. 3a), while $48 \%$ (97/200) did not have these verandas.

It was also observed that other than these verandas (Fig. 3a), most houses had additional peri-domestic spaces where members congregated. Of the 103 that had verandas, 69 (67\%) also had other active peri-domestic spaces, of which 23 were built-up structures and 46, were non-built up. These structures all had at least physical roofing, and $70 \%$ of them also had no wall. Two thirds of the built-up structures were used as outdoor kitchens (60\% used for cooking) as shown in Fig. 3b. Many of the non-built structures $(63 \%)$ were sited under trees (Fig. 3c), while 35\% were in open spaces. The peri-domestic spaces were used for multiple activities, e.g. cooking, eating, socializing among others.

Of 97 houses that did not have veranda extensions, 91 (93.8\%) had active peri-domestic spaces, of which 32 had built up structures with roofs, and also walls in one-third of the cases. Of the non-built structures, $42 \%$ were under trees. Common uses of these spaces were similar, i.e. resting, cooking, eating.

\section{Overall collected mosquitoes}

In the dry season, the total number of mosquitoes collected was 4960, including 2604 Culex spp.; 2264 Anopheles gambiae s.l.; 80 Anopheles coustani; 6 An. funestus; 4 Mansonia spp.; and 2 Coquilettidia mosquitoes. Polymererase chain reaction (PCR) was conducted on 81 samples of An. gambiae s.l. to distinguish between sibling species. Of the $90.1 \%(73 / 81)$ successfully amplified in the PCR assays, all (100\%) were identified as An. arabiensis.
In the wet season the total number of mosquitoes collected was 14,303, including 12,224 Culex spp.; 1978 An. gambiae s.l.; 42 An. funestus; 37 Mansonia spp.; 15 Ae. aegypti; 6 An. coustani; and 1 Anopheles pharoensis. No molecular assay was conducted to identify mosquito species in this particular season.

\section{Efficacy of transfluthrin-treated chairs and transfluthrin-treated hessian ribbons against outdoor-biting mosquitoes in the peri-domestic spaces}

Findings on protective efficacy of the two interventions are summarized in Tables 3 and 4. Using two transfluthrin-treated chairs significantly reduced outdoorbiting $A n$. arabiensis mosquitoes by $76 \%$ (Relative rate $(\mathrm{RR})=0.24,95 \%$ confidence interval, CI 0.19-0.29, $P<0.001)$ and by $85 \%(\mathrm{RR}=0.15,95 \%$ CI $0.12-0.18$, $P<0.001)$ in dry and wet seasons, respectively. Using one transfluthrin-treated chair also significantly reduced $A n$. arabiensis mosquitoes, in this case by $70 \%(\mathrm{RR}=0.30$, 95\% CI $0.25-0.37, P<0.001)$ and by $75 \%(R R=0.25$, 95\% CI $0.20-0.31, P<0.001)$ in dry and wet seasons. When the densities of Culex mosquitoes were assessed, both the two-chair and one-chair interventions significantly reduced outdoor-biting, achieving $52 \%(\mathrm{RR}=0.48$, CI $0.37-0.63, P<0.001)$ and $58 \%(\mathrm{RR}=0.42,95 \% \mathrm{CI}$ $0.31-0.56, P<0.001)$ protection, in dry and wet seasons, respectively. In the wet season, both the two-chair and one-chair interventions significantly reduced outdoorbiting, achieving $51 \%(\mathrm{RR}=0.49, \mathrm{CI} 0.43-0.56, P<0.001)$
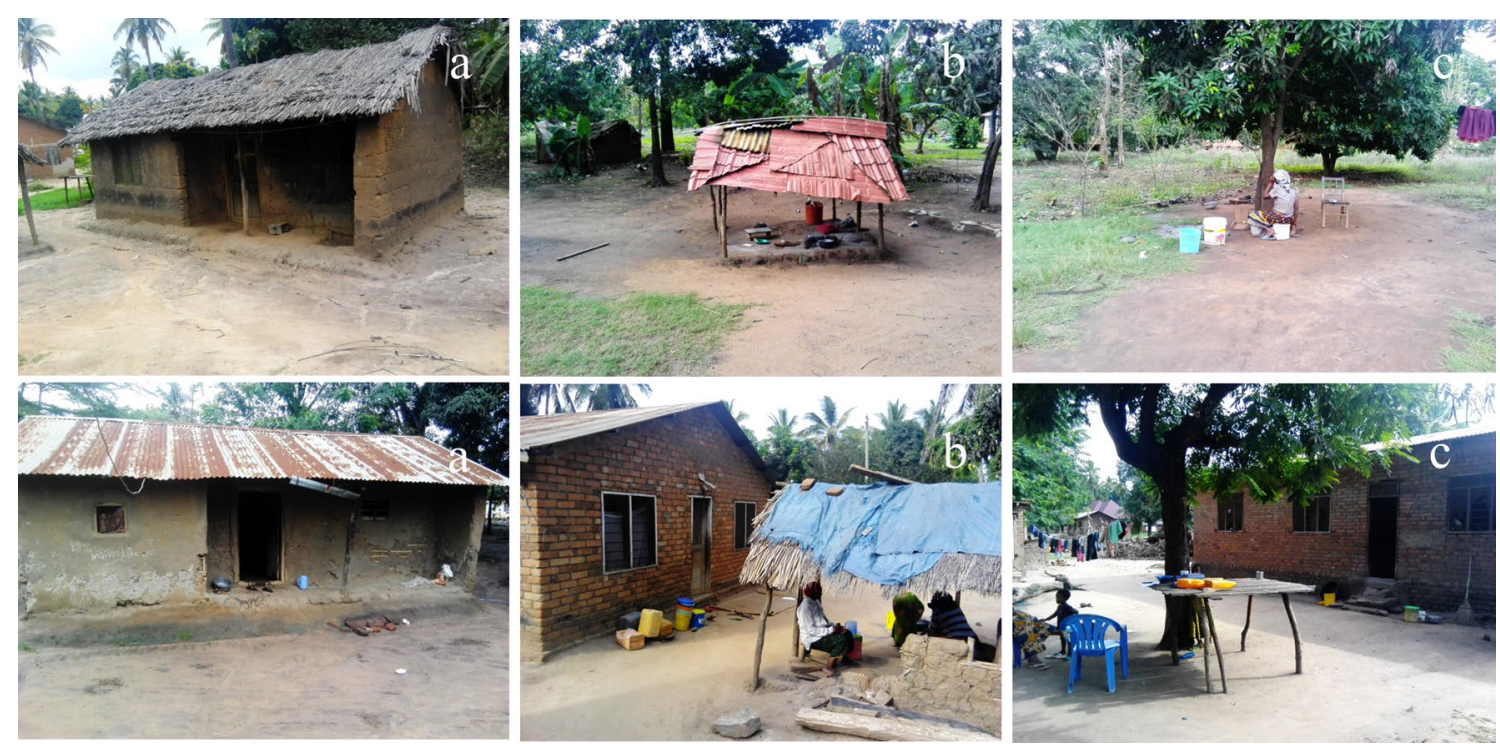

Fig. 3 Illustration of houses with veranda extension physically characterized during survey (a), houses with built-up peridomestic space away from the main house commonly used for cooking (b) and houses with non-built-up peridomestic space physically characterized as under the tree (c) 
Table 3 Comparison of nightly outdoor biting per person between houses with or without transfluthrin-treated chairs or ribbons (dry season)

\begin{tabular}{|c|c|c|c|c|c|c|c|c|}
\hline Settings & Species & Treatment & Nights & $n$ & $\begin{array}{l}\text { Adjusted-mean } \\
(95 \% \mathrm{Cl})\end{array}$ & RR $(95 \% \mathrm{Cl})$ & PP $(95 \% \mathrm{Cl})$ & P-value \\
\hline \multirow{10}{*}{$\begin{array}{l}\text { Outdoor peri- } \\
\text { domestic space }\end{array}$} & \multirow[t]{5}{*}{ Anopheles arabiensis } & Control & 32 & 1056 & 15.05 (12.29-18.44) & 1 & 0 & \\
\hline & & Two TF-chairs & 32 & 273 & $3.61(2.87-4.55)$ & $0.24(0.19-0.29)$ & $0.76(071-0.80)$ & $<0.001$ \\
\hline & & TF-treated ribbon & 32 & 211 & $2.96(2.33-3.75)$ & $0.19(0.16-0.24)$ & $0.81(0.75-0.84)$ & $<0.001$ \\
\hline & & Control & 28 & 910 & $14.86(12.07-18.30)$ & 1 & 0 & \\
\hline & & $\begin{array}{l}\text { One TF-treated } \\
\text { chair }\end{array}$ & 28 & 290 & $4.54(3.60-5.73)$ & $0.30(0.25-0.37)$ & $0.70(0.62-0.75)$ & $<0.001$ \\
\hline & \multirow[t]{5}{*}{ Culex spp. } & Control & 32 & 889 & $10.52(7.98-13.86)$ & 1 & 0 & \\
\hline & & Two TF-chairs & 32 & 426 & $5.12(3.84-6.83)$ & $0.48(0.37-0.63)$ & $0.52(0.36-0.63)$ & $<0.001$ \\
\hline & & TF-treated ribbon & 32 & 299 & $3.43(2.55-4.61)$ & $0.32(0.24-0.43)$ & $0.68(0.57-0.75)$ & $<0.001$ \\
\hline & & Control & 28 & 744 & $9.99(7.43-13.44)$ & 1 & 0 & \\
\hline & & $\begin{array}{l}\text { One TF-treated } \\
\text { chair }\end{array}$ & 28 & 335 & $4.20(3.07-5.75)$ & $0.42(0.31-0.56)$ & $0.58(0.43-0.68)$ & $<0.001$ \\
\hline \multirow{4}{*}{$\begin{array}{l}\text { Inside outdoor } \\
\text { kitchen enclosure }\end{array}$} & \multirow[t]{2}{*}{ Anopheles arabiensis } & Control & 25 & 152 & $1.17(0.56-2.44)$ & 1 & & \\
\hline & & TF-sisal ribbon & 25 & 113 & $0.56(0.26-1.22)$ & $0.57(0.32-1.03)$ & $\begin{array}{l}0.43(-0.03 \text { to } \\
0.67)\end{array}$ & 0.065 \\
\hline & \multirow[t]{2}{*}{ Culex spp. } & Control & 25 & 288 & $2.37(1.35-4.17)$ & 1 & 0 & \\
\hline & & TF-sisal ribbon & 25 & 89 & $0.56(0.29-1.06)$ & $0.23(0.12-0.43)$ & $0.77(0.56-0.87)$ & $<0.001$ \\
\hline
\end{tabular}

$n$ total number of mosquito collected, $\mathrm{Cl}$ confidence interval, $P P$ percentage protection, $R R$ relative rate, $T F$ transfluthrin, 1 and 0 references

Table 4 Comparison of nightly outdoor biting per person between houses with or without transfluthrin-treated chairs or ribbons (wet season)

\begin{tabular}{|c|c|c|c|c|c|c|c|c|}
\hline Settings & Species & Treatment & Nights & $\mathbf{n}$ & $\begin{array}{l}\text { Adjusted-mean } \\
(95 \% \mathrm{Cl})\end{array}$ & $\mathrm{RR}(95 \% \mathrm{Cl})$ & $\mathrm{PP}(95 \% \mathrm{Cl})$ & P-value \\
\hline \multirow{8}{*}{$\begin{array}{l}\text { Outdoor peri-domes- } \\
\text { tic space }\end{array}$} & \multirow[t]{4}{*}{ Anopheles arabiensis } & Control & 32 & 1116 & $5.71(4.89-6.67)$ & 1 & 0 & \\
\hline & & One TF-chair & 32 & 308 & $1.42(1.17-1.72)$ & $0.25(0.20-0.31)$ & $0.75(0.69-0.79)$ & $<0.001$ \\
\hline & & Two TF-chairs & 32 & 189 & $0.86(0.69-1.07)$ & $0.15(0.12-0.18)$ & $0.85(0.81-0.88)$ & $<0.001$ \\
\hline & & TF-treated ribbon & 32 & 273 & $1.32(1.08-1.60)$ & $0.23(0.18-0.28)$ & $0.77(0.71-0.81)$ & $<0.001$ \\
\hline & \multirow[t]{4}{*}{ Culex spp. } & Control & 32 & 4142 & $21.78(18.11-26.18)$ & 1 & 0 & \\
\hline & & One TF-chair & 32 & 2598 & $13.17(10.93-15.86)$ & $0.60(0.53-0.68)$ & $0.40(0.31-0.47)$ & $<0.001$ \\
\hline & & Two TF-chairs & 32 & 2216 & $10.68(8.85-12.87)$ & $0.49(0.43-0.56)$ & $0.51(0.44-0.57)$ & $<0.001$ \\
\hline & & TF-treated ribbon & 32 & 2794 & $13.93(11.56-16.78)$ & $0.64(0.56-0.72)$ & $0.36(0.27-0.44)$ & $<0.001$ \\
\hline \multirow{4}{*}{$\begin{array}{l}\text { Inside outdoor kitchen } \\
\text { enclosure }\end{array}$} & \multirow[t]{2}{*}{ Anopheles arabiensis } & Control & 32 & 68 & Low catches & & & \\
\hline & & TF-sisal ribbon & 32 & 24 & Low catches & & & \\
\hline & \multirow[t]{2}{*}{ Culex spp. } & Control & 32 & 302 & $0.49(0.31-0.78)$ & 1 & 0 & \\
\hline & & TF-sisal ribbon & 32 & 172 & $0.26(0.15-0.43)$ & $0.52(0.32-0.86)$ & $0.48(0.13-0.67)$ & 0.011 \\
\hline
\end{tabular}

$n$ total number of mosquito collected, $C l$ confidence interval, $P P$ percentage protection, $R R$ relative rate, $T F$ transfluthrin, 1 and 0 references

and $40 \% \quad(\mathrm{RR}=0.60, \quad 95 \% \quad \mathrm{CI} \quad 0.53-0.68, \quad P<0.001)$ protection.

Fitting the transfluthrin-treated hessian ribbons around the outdoor kitchens reduced outdoor-biting An. arabiensis by $81 \%$ in the area immediately outside this kitchen enclosure $(\mathrm{RR}=0.19,95 \% \mathrm{CI}$ $0.16-0.24, P<0.001)$, and by $43 \%(\mathrm{RR}=0.57$, CI $0.32-$ 1.03, $P=0.065)$ inside the enclosures in the dry season. In the wet season, transfluthrin-treated hessian ribbons reduced outdoor-biting An. arabiensis by $77 \%$ in the area immediately outside this kitchen enclosure $(\mathrm{RR}=0.23$, 95\% CI $0.18-0.28, P<0.001)$. The ribbons also reduced outdoor-biting Culex by $68 \%(\mathrm{RR}=0.32$, CI $0.24-0.43, P<0.001)$ near the enclosures and by $77 \%$ $(\mathrm{RR}=0.23, \mathrm{CI} 0.12-0.43, P<0.001)$ within the enclosures in the dry season. In the wet season, the ribbons also reduced outdoor-biting Culex by $36 \%(\mathrm{RR}=0.64$, CI $0.56-0.72, P<0.001)$ near the enclosures and by 
$48 \%(\mathrm{RR}=0.52, \mathrm{CI} 0.32-0.86, P<0.001)$ within the enclosures.

\section{Mortality of field-collected or laboratory-reared mosquitoes exposed to transfluthrin-treated chairs}

Findings on induced mortality of mosquitoes exposed to transfluthrin-treated chairs are summarized in Table 5. When field-collected An. arabiensis females and $A n$. funestus females were exposed underneath the transfluthrin-treated chairs, $99.4 \%$ and $100 \%$ of them died within $24 \mathrm{~h}$, respectively. All (100\%) of the laboratoryreared An. arabiensis or laboratory-reared Ae. aegypti mosquitoes exposed also died when exposed underneath the transfluthrin-treated chairs. Mortality of the mosquitoes exposed to untreated chairs however remained low (5.2\% for field-collected An. arabiensis, $0.0 \%$ for field-collected An. funestus, 0.1\% for laboratory-reared An. arabiensis and $1.1 \%$ for laboratory-reared Ae. aegypti).

Insecticide resistance status of mosquitoes in a study area Results of the WHO resistance tests are summarized in Table 6. The field populations of $A n$. arabiensis were fully susceptible to bendiocarb (100\% mortality), pirimiphos methyl (100\% mortality) and DDT (98.8\% mortality). However, they were resistant to both permethrin $(94.7 \%$ mortality) and deltamethrin (80.3\% mortality).

\section{Discussion}

Several studies in tropical settings have documented that many people stay active outdoors in early evenings before they go indoors and then sleep under bed nets $[14,16,17]$. Those studies also characterized the actual activities that people were involved in outdoors. To our knowledge, this current study is the first to characterize the peri-domestic spaces used by household members in a malaria-endemic setting for various outdoor activities.

The key finding was that most houses had active peridomestic spaces (veranda extensions, open general areas and makeshift kitchens) where household members performed different activities, usually unprotected from potentially-infectious mosquitoes before they went indoors. In some of the peri-domestic spaces, residents constructed structures for cooking, eating and socializing, but these too were often open and not protective against mosquito bites (Fig. 3b).

The study also demonstrated that the two simple interventions evaluated, i.e. transfluthrin-emanating chairs and ribbons both considerably reduced outdoor-biting by the important residual malaria vector, $A n$. arabiensis. Furthermore, mosquitoes exposed to the chairs were killed rapidly, indicating that the interventions could offer not just personal or household protection, but also communal protection through mass killing effect, by reducing mosquito density, survival and malaria sporozoite infection prevalence [37].

Table 5 Comparison of induced mortality to mosquitoes exposed to house with or without transfluthrin-treated chairs

\begin{tabular}{|c|c|c|c|c|c|c|}
\hline Settings & Species & Treatment & Days & Exposed & Dead $24 \mathrm{~h}$ & Mortality (\%) \\
\hline \multirow[t]{4}{*}{ Wild mosquitoes } & \multirow[t]{2}{*}{ Anopheles arabiensis } & Control & 10 & 1142 & 60 & 5.2 \\
\hline & & TF-treated chair & 10 & 1140 & 1134 & 99.4 \\
\hline & \multirow[t]{2}{*}{ Anopheles funestus } & Control & 5 & 490 & 0 & 0 \\
\hline & & TF-treated chair & 5 & 490 & 490 & 100 \\
\hline \multirow[t]{4}{*}{ Lab-reared mosquitoes } & \multirow[t]{2}{*}{ Anopheles arabiensis } & Control & 9 & 860 & 10 & 1.1 \\
\hline & & TF-treated chair & 9 & 860 & 860 & 100 \\
\hline & \multirow[t]{2}{*}{ Aedes aegypti } & Control & 9 & 900 & 3 & 0.3 \\
\hline & & TF-treated chair & 9 & 900 & 900 & 100 \\
\hline
\end{tabular}

TF transfluthrin

Table 6 Show insecticide resistant status in Anopheles arabiensis mosquitoes to difference insecticides at Lupiro village

\begin{tabular}{llll}
\hline Insecticide tested & Mosquito species tested & Percentage mortality (\%) & Resistance status \\
\hline Bendiocarb & Anopheles arabiensis & 100 & Susceptible \\
Pirimiphos-methyl & Anopheles arabiensis & 100 & Susceptible \\
DDT & Anopheles arabiensis & 98.8 & Susceptible \\
Permethrin & Anopheles arabiensis & 94.7 & Resistant (after confirmation) \\
Deltamethrin & Anopheles arabiensis & 80.3 & Resistant \\
\hline
\end{tabular}


More than half the households surveyed had veranda extensions with roofed enclosures, mostly used for resting, cooking and eating. All these structures provide opportunities for mounting simple interventions in these spaces such as physical screening and complementary chemical measures like these transfluthrin emanator formats and turning them into mosquito proof areas as they are predominantly used for early-evening human activities, notably resting, cooking and eating.

The findings that transfluthrin-emanating chairs provided useful levels of protection against An. arabiensis and Culex spp. corroborate previous observations with other prototypes in outdoor bars [33]. Even though the prototype (chair) used in this study differs to those used in previous studies (decoration) [33], it emphasizes the potential of these technologies for outdoor protection in such communities. Further research should therefore focus on improvement of the prototypes and optimization of the treatments.

Outdoor kitchens were commonly used for cooking in early evening, and were among the commonest constructed spaces identified in households, regardless of whether they had verandas or not. Early-evening cooking within this space coincides with peak hours of mosquito bites [54], amplifying the likelihood of malaria transmission in these spaces. In this study, the high levels of protection provided against An. arabiensis by the repellent-treated hessian ribbons around these outdoor kitchens is, therefore, encouraging and consistent with previous studies [34], which demonstrated that transfluthrin-treated hessian ribbons protected non-users against $A n$. arabiensis sitting within radius of 5 metres. More recently, transfluthrin-treated ribbons fitted to the eaves of houses prevented both indoor and outdoorbiting mosquitoes [31, 36, 37]. Since the increase in temperature also increases the rate of transfluthrin evaporation, the cooking activity within the kitchen may have increased insecticidal activity of transfluthrin. The effect of temperature was also well described by Ogoma et al. [34]. This high level of protection provided against $A n$. arabiensis by the ribbons may have been positively influenced by cooking activities within these enclosures.

In addition to the substantial protection against $A n$. arabiensis demonstrated in the areas immediately outside the ribbon-fitted kitchen, the catches by CDC light traps placed within the kitchens are reduced, albeit more modestly. This modest reduction may be due to the use of CDC light traps in these open spaces, which may have resulted in exaggerated catches of mosquitoes attracted by the light bulb in the traps. It may also be due to the smoke produced from these kitchens, which may have confounded the results observed on An. arabiensis. Interestingly, this emanator prototype provided much more satisfactory protection against nuisance-causing Culex spp. within the kitchens based on the same CDC light trap catches. It is not clear why such significant reductions observed for Culex spp. were not observed for An. arabiensis, but it is nevertheless encouraging that reduced Culex spp. densities should motivate user acceptance. It is also encouraging that these observations are also broadly consistent with previous studies [31,32] demonstrating that outdoor use of transfluthrin-treated hessian provided more than $90 \%$ protection against both An. gambiae s.l. and Culex spp. mosquitoes [31, 32].

Pyrethroid-treated nets divert host-seeking mosquitoes from humans or kill the mosquitoes attempting to feed on the protected persons $[55,56]$. With these modes of action, pyrethroid-treated nets not only provide personal protection (to users), but also communal protection (to both users and non-users) by suppressing vectors population through the mass killing effect $[57,58]$. Transfluthrin, used to treat the hessian mats fitted underneath the chairs induced high mortality on caged mosquitoes exposed underneath the experimental chairs $(100 \%$ in most cases). This implies that the chairs may not only provide personal protection, but also community benefit through mass-killing of mosquitoes, even without the mosquitoes making contact with treated surfaces. This effect was particularly important since the field-collected mosquitoes were from villages where Anopheles populations were pyrethroid-resistant (Table 6).

To date, there is no literature which explains the best exposure time for mosquitoes in transfluthrin-treated material that achieves 50\% mortality. However, Ogoma et al. [38, 59] demonstrated that even short exposures of 15 min reduced mosquito blood feeding significantly. In this current study, the selection of exposure time was based on what period a particular mosquito species is active. For the day-biting mosquitoes, a day-time exposure was selected and for night-time biting species a night-time exposure was selected.

Even though excito-repellency effects maximize person protection by chasing mosquitoes away, it may attenuate more important mass killing effects by deterring mosquitoes from making fatal contact with lethal doses of the repellent insecticide itself or with complementary solid-phase insecticides applied as LLINs or IRS [6062]. However, these observations of mortality amongst wild malaria vectors exposed to transfluthrin suggest that mass population suppression could be achieved even without mosquitoes necessarily touching treated surfaces. It is also encouraging that Ogoma et al. [34] demonstrated that transfluthrin-treated emanator provided more than $90 \%$ biting reduction against $A n$. arabiensis without any obvious diversion to non-users [34]. Another study by Ogoma et al. [38] also observed that 


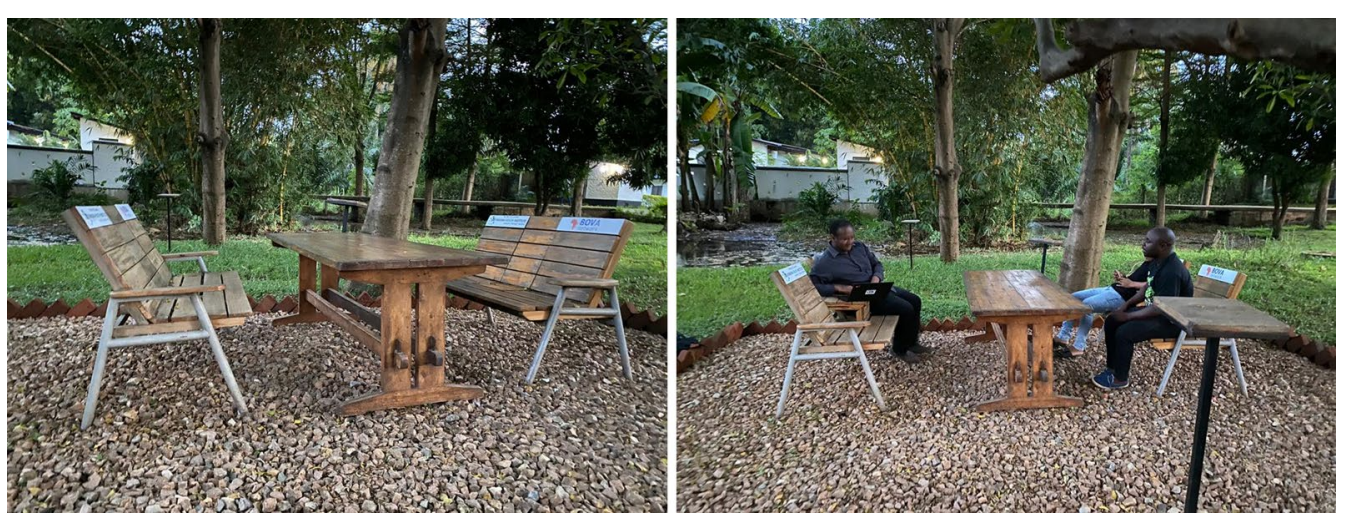

Fig. 4 Picture of the first mosquito-free zone established at Ifakara Health Institute in January 2020. The chairs have transfluthrin-treated hessian mats underneath, but are layered with plastic sheeting to prevent rainfall and user contact

transfluthrin-treated coils could protect non-users within $20 \mathrm{~m}$ radius. More recently, Mwanga et al. demonstrated that transfluthrin-treated ribbons fitted to the eave gaps of houses protected volunteers both inside and outside the houses [37].

The spread of pyrethroid resistance in malaria vectors clearly compromises ongoing control and elimination efforts [63-65]. This is a key concern since transfluthrin is also a pyrethroid. It is however encouraging that transfluthrin-based interventions tested here killed almost $100 \%$ of the wild-caught An. arabiensis and An. funestus exposed to emanated vapour from the chairs, even though local populations of both species are clearly resistant to the conventional solid-phase pyrethoids used for LLINs and IRS [41]. It was surprising that transfluthrin, a pyrethroid, was still efficacious against pyrethroid-resistant malaria mosquitoes. However, given that there is no standard resistance test against transfluthrin, it is difficult to explain as to why transfluthrin demonstrated such high mortality. One possible explanation is the long exposure of up to $12 \mathrm{~h}$ underneath the transfluthrin-treated chairs. Tests with PBO have established that the resistance in this area is of metabolic nature, thus it may be helpful that these new interventions are considered as complementary to other interventions, e.g. IRS or LLINs using active ingredients not affected by this form of resistance.

Usage of chairs cut across different settings, such as normal households, public places, official surroundings used for resting after working hours (Fig. 4). Based on this information, the use of transfluthrin-treated chairs may be rolled out as a complementary vector control strategy even during dengue fever outbreak.

One important limitation of this study was that caged mosquitoes were placed underneath the transfluthrintreated chairs for $12 \mathrm{~h}$. This long-time exposure may well greatly exceed true exposure levels in the field, where mosquitoes can freely fly around and way upon encountering airborne insecticide. Nonetheless, since transfluthrin effects are vapor-mediated, this initial attempt to quantify possible lethal modes of action is encouraging and offers a basis for future improvements in study designs for developing and evaluating these technologies.

\section{Conclusions}

Most houses in this rural African context had well-used peri-domestic spaces (veranda extensions, makeshift kitchens and completely open spaces) where members performed different activities before bed time, usually unprotected from potentially-infectious mosquitoes before they went indoors. Both the transfluthrin-emanating chairs and ribbons reduced outdoor exposure to biting malaria vectors in these peri-domestic spaces and also caused significant mortality of caged, field collected malaria vector mosquitoes. The two emanator prototypes still require additional improvements, optimizations and assessments in future studies, but they could potentially constitute new options for outdoor malaria prevention to complement LLINs and IRS in areas where peri-domestic human activities are common.

\begin{abstract}
Abbreviations
CDC: Center of disease control; Cl: Confidence interval; GLMM: Generalized linear mixed effects model; IRS: Indoor residual spraying; IHI: Ifakara Health Institute; IRB: Institutional Review Board; LLINs: Long-lasting insecticidal nets; NIMR: National Institute for Medical Research; PCR: Polymerase chain reaction; RR: Relative rate
\end{abstract}

\section{Acknowledgements}

We sincerely thank volunteers and technicians involved in this study. Our appreciations also go to laboratory staff at Ifakara Health Institute, Mr. Said Abbasi and Mr. Francis Tumbo who conducted PCR analysis of mosquito samples.

Authors' contributions

JPM, GFK and FOO conceived the idea, helped to obtain the funds, designed and conducted experiments, analysed the data and drafted the manuscript; 
MF helped in the design of the peri-domestic survey, edited and revised the manuscript; HSN helped on data analysis and edited the manuscript; PGM helped in conducting WHO standard susceptibility test on mosquitoes, edited and revised the manuscript; FOO and GFK edited and revised the manuscript. All authors read and approved the final manuscript.

\section{Funding}

The study was funded by Biotechnology and Biological Sciences Research Council (BBSRC) through Building Out Vector-borne diseases in sub-Saharan Africa: the BOVA Network (Grant number: BOVA007). FOO was also funded by Wellcome Trust Intermediate Fellowship in Public Health and Tropical Medicine (Grant number:WT102350/Z/13/Z) and the Howard Hughes Medical Institute (HHMI)-Gates International Scholarship (Grant number: OPP1099295),

\section{Availability of data and materials}

Not applicable.

\section{Ethics approval and consent to participate}

The study was approved by the Institute Review Board of Ifakara Health Institute IHI/IRB/No: 02-2019 and Medical Research Coordinated Committee of the National Institute for Medical Research of the United Republic of Tanzania (NIMR/HQ/R.8a/Nol.1X/3152). All study participants were recruited after signing informed consent forms.

\section{Consent for publication}

This manuscript has been approved for publication by Institute for Medical Research of the United Republic of Tanzania (NIMR/HQ/P.12VOLXXIX/39).

\section{Competing interests}

The authors declare that they have no competing interests.

\begin{abstract}
Author details
${ }^{1}$ Environmental Health and Ecological Sciences Department, Ifakara Health Institute, Morogoro, United Republic of Tanzania. ${ }^{2}$ School of Life Science and Biotechnology, Nelson Mandela African Institution of Science and Technology, Arusha, United Republic of Tanzania. ${ }^{3}$ School of Public Health, University of the Witwatersrand, Parktown, Johannesburg, Republic of South Africa. ${ }^{4}$ Department of Vector Biology, Liverpool School of Tropical Medicine, Liverpool, UK. ${ }^{5}$ Institute of Biodiversity, Animal Health and Comparative Medicine, University of Glasgow, Glasgow, UK.
\end{abstract}

Received: 22 December 2019 Accepted: 2 March 2020

Published online: 10 March 2020

\section{References}

1. WHO. World malaria report 2015. Geneva: World Health Organization; 2015.

2. Bhatt S, Weiss DJ, Cameron E, Bisanzio D, Mappin B, Dalrymple U, et al. The effect of malaria control on Plasmodium falciparum in Africa between 2000 and 2015. Nature. 2015;526:207-11.

3. WHO. World malaria report 2018. Geneva: World Health Organization; 2018.

4. WHO. Word malaria report 2017. Geneva: World Health Organization; 2017.

5. WHO. World malaria report 2019. Geneva: World Health Organization; 2019.

6. Noor AM, Kinyoki DK, Mundia CW, Kabaria CW, Mutua JW, Alegana VA et al. The changing risk of Plasmodium falciparum malaria infection in Africa: 2000-10: a spatial and temporal analysis of transmission intensity. Lancet. 2014;383:1739-47.

7. O'Meara WP, Mangeni JN, Steketee R, Greenwood B. Changes in the burden of malaria in sub-Saharan Africa. Lancet Infect Dis. 2010;10:545-55.

8. Steketee RW, Campbell CC. Impact of national malaria control scale-up programmes in Africa: magnitude and attribution of effects. Malar J. 2010;9:299.

9. Govella NJ, Ferguson H. Why use of interventions targeting outdoor biting mosquitoes will be necessary to achieve malaria elimination. Front Physiol. 2012;3:199.
10. Russell TL, Govella NJ, Azizi S, Drakeley CJ, Kachur SP, Killeen GF. Increased proportions of outdoor feeding among residual malaria vector populations following increased use of insecticide-treated nets in rural Tanzania. Malar J. 2011;10:80.

11. Durnez L, Coosemans M. Residual transmission of malaria: an old issue for new approaches. In: Manguin S, editor. Anopheles mosquitoes: new insight into malaria vectors. London: IntechOpen; 2013.

12. Sherrard-Smith E, Skarp JE, Beale AD, Fornadel C, Norris LC, Moore SJ, et al. Mosquito feeding behavior and how it influences residual malaria transmission across Africa. Proc Natl Acad Sci USA. 2019;116:15086-95.

13. WHO. Global technical strategy for malaria 2016-2030. Geneva: World Health Organization; 2015.

14. Monroe A, Moore S, Koenker H, Lynch M, Ricotta E. Measuring and characterizing night time human behaviour as it relates to residual malaria transmission in sub-Saharan Africa: a review of the published literature. Malar J. 2019;18:6.

15. Monroe A, Asamoah O, Lam Y, Koenker H, Psychas P, Lynch M, et al. Outdoor-sleeping and other night-time activities in northern Ghana: implications for residual transmission and malaria prevention. Malar J. 2015;14:35.

16. Moshi IR, Ngowo H, Dillip A, Msellemu D, Madumla EP, Okumu FO, et al. Community perceptions on outdoor malaria transmission in Kilombero Valley, Southern Tanzania. Malar J. 2017;16:274.

17. Finda MF, Moshi IR, Monroe A, Limwagu AJ, Nyoni AP, Swai JK, et al. Linking human behaviours and malaria vector biting risk in south-eastern Tanzania. PLoS ONE. 2019;14:e0217414.

18. Williams YA, Tusting LS, Hocini S, Graves PM, Killeen GF, Kleinschmidt I, et al. Expanding the vector control toolbox for malaria elimination: a systematic review of the evidence. Adv Parasitol. 2018;99:345-79.

19. Homan T, Hiscox A, Mweresa CK, Masiga D, Mukabana WR, Oria P, et al. The effect of mass mosquito trapping on malaria transmission and disease burden (SolarMal): a stepped-wedge cluster-randomised trial. Lancet. 2016;388:1193-201.

20. Okumu FO, Govella NJ, Moore SJ, Chitnis N, Killeen GF. Potential benefits, limitations and target product-profiles of odor-baited mosquito traps for malaria control in Africa. PLoS ONE. 2010;5:e11573.

21. Müller GC, Beier JC, Traore SF, Toure MB, Traore MM, Bah S, et al. Successful field trial of attractive toxic sugar bait (ATSB) plant-spraying methods against malaria vectors in the Anopheles gambiae complex in Mali, West Africa. Malar J. 2010;9:210.

22. Crawshaw AF, Maung TM, Shafique M, Sint N, Nicholas S, Li MS, et al. Acceptability of insecticide-treated clothing for malaria prevention among migrant rubber tappers in Myanmar: a cluster-randomized noninferiority crossover trial. Malar J. 2017;16:92.

23. Rowland M, Durrani N, Hewitt S, Mohammed N, Bouma M, Carneiro I, et al. Permethrin-treated chaddars and top-sheets: appropriate technology for protection against malaria in Afghanistan and other complex emergencies. Trans R Soc Trop Med Hyg. 1999;93:465-72.

24. Rowland M, Durrani N, Kenward M, Mohammed N, Urahman H, Hewitt S. Control of malaria in Pakistan by applying deltamethrin insecticide to cattle: a community-randomised trial. Lancet. 2001;357:1837-41.

25. Gupta RK, Rutledge LC. Role of repellents in vector control and disease prevention. Am J Trop Med Hyg. 1994;50:82-6.

26. Moore SJ, Davies CR, Hill N, Cameron MM. Are mosquitoes diverted from repellent-using individuals to non-users? Results of a field study in Bolivia. Trop Med Int Health. 2007;2:532-9.

27. Maia MF, Kliner M, Richardson M, Lengeler C, Moore SJ. Mosquito repellents for malaria prevention. Cochrane Database Syst Rev. 2018;2:CD011595.

28. Gryseels C, Uk S, Sluydts V, Durnez L, Phoeuk P, Suon S, et al. Factors influencing the use of topical repellents: implications for the effectiveness of malaria elimination strategies. Sci Rep. 2015;5:16847.

29. Makungu C, Stephen S, Kumburu S, Govella NJ, Dongus S, Hildon ZJ-L, et al. Informing new or improved vector control tools for reducing the malaria burden in Tanzania: a qualitative exploration of perceptions of mosquitoes and methods for their control among the residents of Dar es Salaam. Malar J. 2017;16:410.

30. Sangoro O, Kelly AH, Mtali S, Moore SJ. Feasibility of repellent use in a context of increasing outdoor transmission: a qualitative study in rural Tanzania. Malar J. 2014;13:347. 
31. Ogoma SB, Ngonyani H, Simfukwe ET, Mseka A, Moore J, Killeen GF. Spatial repellency of transfluthrin-treated hessian strips against laboratoryreared Anopheles arabiensis mosquitoes in a semi-field tunnel cage. Parasit Vectors. 2012;5:54.

32. Govella NJ, Ogoma SB, Paliga J, Chaki PP, Killeen G. Impregnating hessian strips with the volatile pyrethroid transfluthrin prevents outdoor exposure to vectors of malaria and lymphatic filariasis in urban Dar es Salaam, Tanzania. Parasit Vectors. 2015;8:322.

33. Masalu JP, Finda M, Okumu FO, Minja EG, Mmbando AS, Sikulu-Lord MT, et al. Efficacy and user acceptability of transfluthrin-treated sisal and hessian decorations for protecting against mosquito bites in outdoor bars. Parasit Vectors. 2017;10:197.

34. Ogoma SB, Mmando AS, Swai JK, Horstmann S, Malone D, Killeen GF. A low technology emanator treated with the volatile pyrethroid transfluthrin confers long term protection against outdoor biting vectors of lymphatic filariasis, arboviruses and malaria. PLoS Negl Trop Dis. 2017;11:e0005455.

35. Achee NL, Bangs MJ, Farlow R, Killeen GF, Lindsay S, Logan JG, et al. Spatial repellents: from discovery and development to evidence-based validation. Malar J. 2012;11:164.

36. Mmbando AS, Ngowo H, Limwagu A, Kilalangongono M, Kifungo K, Okumu FO. Eave ribbons treated with the spatial repellent, transfluthrin, can effectively protect against indoor-biting and outdoor-biting malaria mosquitoes. Malar J. 2018;17:368.

37. Mwanga EP, Mmbando AS, Mrosso PC, Stica C, Mapua SA, Finda MF, et al. Eave ribbons treated with transfluthrin can protect both users and nonusers against malaria vectors. Malar J. 2019;18:314.

38. Ogoma SB, Ngonyani H, Simfukwe ET, Mseka A, Moore J, Maia MF, et al. The mode of action of spatial repellents and their impact on vectorial capacity of Anopheles gambiae sensu stricto. PLoS ONE. 2014;9:e110433.

39. Tanzania Meteorological Agency. http://www.meteo.go.tz/. Accessed 15 Oct 2019.

40. World Weather Online. https://www.worldweatheronline.com/ulang a-weather/moro. Accessed 15 Oct 2019.

41. Kaindoa EW, Matowo NS, Ngowo HS, Mkandawile G, Mmbando A, Finda $\mathrm{M}$, et al. Interventions that effectively target Anopheles funestus mosquitoes could significantly improve control of persistent malaria transmission in south-eastern Tanzania. PLoS ONE. 2017;12:e0177807.

42. Lwetoijera DW, Harris C, Kiware SS, Dongus S, Devine GJ, McCall PJ, Majambere S. Increasing role of Anopheles funestus and Anopheles arabiensis in malaria transmission in the Kilombero Valley, Tanzania. Malar J. 2014;13:331.

43. Matowo NS, Munhenga G, Tanner M, Coetzee M, Feringa WF, Ngowo $\mathrm{HS}$, et al. Fine-scale spatial and temporal heterogeneities in insecticide resistance profiles of the malaria vector, Anopheles arabiensis in rural south-eastern Tanzania. Wellcome Open Res. 2017;2:96.

44. Renggli S, Mandike R, Kramer K, Patrick F, Brown NJ, McElroy PD, et al. Design, implementation and evaluation of a national campaign to deliver 18 million free long-lasting insecticidal nets to uncovered sleeping spaces in Tanzania. Malar J. 2013;12:85

45. KoBoToolbox: Simple, robust and powerful tools for data collection. https ://www.kobotoolbox.org/. Accessed 17 Oct 2019.

46. Limwagu AJ, Kaindoa EW, Ngowo HS, Hape E, Finda M, Mkandawile G, et al. Using a miniaturized double-net trap (DN-Mini) to assess relationships between indoor-outdoor biting preferences and physiological ages of two malaria vectors, Anopheles arabiensis and Anopheles funestus. Malar J. 2019;18:282.

47. Sudia WD, Chamberlain RW. Battery-operated light trap, an improved model. J Am Mosq Control Assoc. 1988;4:536-8.

48. Gillies M, Coetzee M. A supplement to the Anophelinae of Africa South of the Sahara. Publ S Afr Inst Med Res. 1987;55:1-143.

49. Kahamba NF, Limwagu AJ, Mapua SA, Msugupakulya BJ, Msaky DS, Kaindoa EW, et al. Habitat characteristics and insecticide susceptibility of Aedes aegypti in the Ifakara area, south-eastern Tanzania. Parasit Vectors. 2020;13:53.
50. Masalu JP, Okumu FO, Mmbando AS, Sikulu-Lord MT, Ogoma SB. Potential benefits of combining transfluthrin-treated sisal products and longlasting insecticidal nets for controlling indoor-biting malaria vectors. Parasit Vectors. 2018;11:231.

51. WHO. Test procedures for insecticide resistance monitoring in malaria vector mosquitoes. 2nd ed. Geneva: World Health Organization; 2018.

52. R Core Team. R: a language and environment for statistical computing. Vienna: R Foundation for Statistical Computing; 2012. http://www.R-proje ct.org. 2018.

53. Bates D, Mächler M, Bolker B, Walker S. Fitting linear mixed-effects models Usinglme4. J Stat Soft. 2015;67:1.

54. Matowo NS, Moore J, Mapua S, Madumla EP, Moshi IR, Kaindoa EW, et al. Using a new odour-baited device to explore options for luring and killing outdoor-biting malaria vectors: a report on design and field evaluation of the Mosquito Landing Box. Parasit Vectors. 2013;6:137.

55. Lindsay SW, Adiamah JH, Miller JE, Armstrong JRM. Pyrethroid-treated bednet effects on mosquitoes of the Anopheles gambiae complex in The Gambia. Med Vet Entomol. 1991;5:477-83.

56. Miller JE, Lindsay SW, Armstrong JRM. Experimental hut trials of bednets impregnated with synthetic pyrethroid or organophosphate insecticide for mosquito control in The Gambia. Med Vet Entomol. 1991;5:465-76.

57. Carnevale P, Robert V, Boudin C, Halna JM, Pazart L, Gazin P, et al. Control of malaria using mosquito nets impregnated with pyrethroids in Burkina Faso. Bull Soc Pathol Exot. 1988;81:832-46.

58. Magesa SM, Wilkes TJ, Mnzava AE, Njunwa KJ, Myamba J, Kivuyo MD, et al. Trial of pyrethroid impregnated bednets in an area of Tanzania holoendemic for malaria. Part 2. Effects on the malaria vector population. Acta Trop. 1991;49:97-108.

59. Ogoma SB, Lorenz LM, Ngonyani H, Sangusangu R, Kitumbukile M, Kilalangongono $\mathrm{M}$, et al. An experimental hut study to quantify the effect of DDT and airborne pyrethroids on entomological parameters of malaria transmission. Malar J. 2014;13:131.

60. Killeen GF, Chitnis N, Moore SJ, Okumu FO. Target product profile choices for intra-domiciliary malaria vector control pesticide products: repel or kill? Malar J. 2011;10:207.

61. Killeen GF, Moore SJ. Target product profiles for protecting against outdoor malaria transmission. Malar J. 2012;11:17.

62. Killeen GF, Seyoum A, Gimnig JE, Stevenson JC, Drakeley CJ, Chitnis N. Made-to-measure malaria vector control strategies: rational design based on insecticide properties and coverage of blood resources for mosquitoes. Malar J. 2014;13:146.

63. Protopopoff N, Mosha JF, Lukole E, Charlwood JD, Wright A, Mwalimu $C D$, et al. Effectiveness of a long-lasting piperonyl butoxide-treated insecticidal net and indoor residual spray interventions, separately and together, against malaria transmitted by pyrethroid-resistant mosquitoes: a cluster, randomised controlled, two-by-two factorial design trial. Lancet. 2018;391:1577-88.

64. Cook J, Tomlinson S, Kleinschmidt I, Donnelly MJ, Akogbeto M, Adechoubou A, et al. Implications of insecticide resistance for malaria vector control with long-lasting insecticidal nets: trends in pyrethroid resistance during a WHO-coordinated multi-country prospective study. Parasit Vectors. 2018;1:550.

65. Tiono AB, Ouédraogo A, Ouattara D, Bougouma EC, Coulibaly S, Diarra A, et al. Efficacy of Olyset Duo, a bednet containing pyriproxyfen and permethrin, versus a permethrin-only net against clinical malaria in an area with highly pyrethroid-resistant vectors in rural Burkina Faso: a cluster-randomised controlled trial. Lancet. 2018;392:569-80.

\section{Publisher's Note}

Springer Nature remains neutral with regard to jurisdictional claims in published maps and institutional affiliations. 